\title{
WAR DIE KONTERREVOLUTION UNVERMEIDLICH? MITTELSCHICHTEN UND MILITÄR IN CHILE*
}

\author{
Von Dieter Nohlen und Otto Boye
}

Am 11. September 1973 beendete der Putsch der Streitkräfte und der Polizei des Landes das sozialistische Experiment in Chile. War das militärische Eingreifen in den chilenischen politischen Prozeß unvermeidlich? Galt der Putsch schlechthin dem Versuch, in Chile den Sozialismus entsprechend dem Programm der Volkseinheit aufzubauen? Oder richtete er sich gegen politische Kräfte und Entwicklungen, deren Entstehen und/oder Erstarken aus der Dynamik des eingeleiteten revolutionären Prozesses resultierten? Um diese für den Inhalt des „Lehrstücks Chile“ entscheidende Frage zu beantworten, wollen wir im folgenden die Gründe des Scheiterns von Salvador Allende untersuchen und uns dabei vor allem der Rolle zuwenden, die die Mittelschichten ${ }^{1}$ und das Militär im politischen Prozeß in Chile spielen, wobei wir namentlich die Jahre 1970 bis 1973 betrachten werden.

\section{Die soziale und politische Konzeption der Volkseinheit}

Offensichtlich hat das Verhalten der Mittelschichten, jener heterogenen, durch ihre mittlere Stellung zwischen Ober- und Unterschicht gekennzeichneten Schichten, welche die soziale Basis der Streitkräfte bilden, Verlauf und schließliches Schicksal des sozialistischen Experiments entscheidend mitbestimmt. Der Militärputsch kann mit einigem Recht als die Konterrevolution der Mittelschichten bezeichnet werden ${ }^{2}$. Um die schwierige Beziehung Volkseinheit - Mittelschichten $\mathrm{zu}$ verstehen, die das Problem des Überlebens der Revolution in sich barg, müssen wir auf die Genesis des linken Parteienbündnisses zu sprechen kommen. Für unseren Zusammenhang sind drei Aspekte bei der Konstitution der Volkseinheit von fundamentaler Bedeutung:

1. Die Volkseinheit wurde als ein Klassenbündnis von Arbeiterschaft und Mittelschichten konzipiert;

2. Die politische Strategie der Volkseinheit folgte nicht dem „wissenschaftlichen Sozialismus", sondern war reformistisch und respektierte folglich das bürgerliche Institutionensystem als Rahmenbedingung revolutionärer Veränderung der chilenischen Gesellschaft;

\footnotetext{
" Vgl. Dieten Nohlen, Sozio-ökonomischer Wandel und Verfassungsreform in Chile 1925-1972, VRU 1973, S. 65.

1 Die Verwendung dieses Schichtungsbegriffs im Plural soll auf die Heterogenität, ja sogar den Antagonismus der unter diese Kategorie einzuordnenden gesellschaftlichen Schichten aufmerksam machen. Zum Konzept "Mittelklasse“ und zur Kritik des modernisierungstheoretischen "Mittelklasse“-Entwicklungsansatzes siehe Rodolfo Stavenhagen, Sieben falsche Thesen über Lateinamerika, in: Andre Gunder Frank, Che Guevara, Mauro Marini, Luis Vitale u. a.: Kritik des bürgerlichen Anti-Imperialismus, Berlin 1969, S. 15-30, S. $23 \mathrm{ff}$. Vgl. auch Ignacio Sotelo, Soziologie Lateinamerikas, Stuttgart 1973, S. $147 \mathrm{ff}$.

2 Diese Formulierung trifft insofern, als die Mittelschichten sich tatsächlich durch die Allende-Regierung, vor allem in ihrer chaotischen Schlußphase, existentiell bedroht fühlten und die Militärs daraus die Legitimität ihres Eingreifens ableiten konnten, so daß der Putsch angesichts der breiten Mittelschichten Chiles anfänglich durchaus eine mehrheitliche Zustimmung der Bevölkerung fand. In seiner Rede zum Jahrestag des Putsches erinnerte General Pinochet an diesen Sachverhalt, als er unterstellte, daß ,die physische Destruktion der Mittelklasse" das Ziel der Volkseinheit gewesen sei (siehe Mercurio, 12. September 1974, S. 8). Die Formulierung erfaßt aber nicht, daß der Putsch vom ehemals ökonomisch und sozial privilegierten Sektor der "Mittelschichten“, also von oligarchischen Gruppen geführt und in seinem sozialen und wirtschaftlichen Inhalt bestimmt wurde. Des weiteren müßte die inzwischen erfolgte Abkehr der unteren und mittleren Mittelschichten von der Militärdiktatur berücksichtigt werden.
} 
3. Das politische Programm der Volkseinheit war in erster Linie anti-oligarchisch und anti-imperialistisch und ging auch in seinem sozialistischen Kern (Verstaatlichungen, staatliche Kontrollen, Planwirtschaft) konform mit den Vorstellungen breiter Sektoren der Mittelschichten hinsichtlich des für Chile als zwingend notwendig angesehenen Entwicklungsweges.

Die Kommunistische Partei Chiles, die die ideologische und strategische Linie der Volkseinheit erarbeitete, war sich des Tatbestandes vollkommen bewußt, daß für den Sieg eines linken Parteienbündnisses bei den Wahlen von 1970 der Beitrag der Mittelschichten ausschlaggebend sein würde. Sie schätzte deren Gewicht in der Sozialstruktur Chiles richtig ein ${ }^{3}$ und zog damit auch die Konsequenz aus den Wahlniederlagen der FRAP (Frente de Acción Popular) von 1958 und 1964, als die Klassenbasis zum Kriterium der Bündnispolitik gemacht wurde und Kommunisten und Sozialisten miteinander koalierten. Die Kommunisten bemühten sich vor allem um die Radikale Partei, die Partei der alten Mittelschichten (freie Berufe), aber auch um kleine unabhängig und sozialdemokratisch orientierte Splittergruppen. Sie hofften des weiteren auf Zuzug aus den Reihen der Christdemokraten, die sie jedoch insgesamt als Bündnispartner ablehnten ${ }^{4}$. Tatsächlich verwirklichte sich in der Koalition von Kommunisten, Sozialisten, Radikalen, Sozialdemokraten, Unabhängiger Volksaktion und Bewegung für die einheitliche Volksaktion (MAPU) ${ }^{5}$ ein Klassenbündnis aus Arbeiterschaft und Mittelschichten. Bereits bei der Konstitution der Volkseinheit betraf die Einbeziehung der Mittelschichten in das linke Parteienbündnis vital die Möglichkeit einer sozialistischen Entwicklung Chiles überhaupt. Ohne die Wählerstimmen, die die Parteien der Mittelschichten der Volkseinheit zutrugen, hätte Allende den ihn am stärksten bedrängenden Konkurrenten, den Kandidaten der politischen Rechten, Jorge Alessandri, nicht bezwingen können, den er nur um 40000 Stimmen übertraf 6 . Wie gering auch der Beitrag der Radikalen insgesamt gewesen sein mag ${ }^{7}$, er war deshalb bedeutend, weil er Allende ermöglichte, im ersten Wahlgang eine relative Mehrheit der Stimmen auf sich zu vereinigen.

3 Zur Sozialgeschichte Chiles siehe den Reader von Hernán Godoy (Hrsg.), Estructura social de Chile, Santiago 1971. Dort findet sich eine umfangreiche Bibliographie. Besonders verweisen möchten wir auf einige Arbeiten von Aníbal Pinto, Carlos Sepúlveda Vergara und Alejandro Foxley, die Dieter Nohlen in: Chile - das sozialistische Experiment, Hamburg 1973, S. $65 \mathrm{ff}$. und passim aufgegriffen hat. Die Konzeption der Kommunistischen Partei geht am besten aus den Reden und Schriften von Luis Corvalán hervor: Luis Corvalán, Camino de victoria, Santiago 1971; Martha Buschmann (Hrsg.), Freiheit für Chile! Reden und Aufsätze von Luis Corvalán 1967-1973, Frankfurt 1973.

$4 \mathrm{Zu}$ erinnern ist hier an die namentlich seit 1969 vom linken Flügel der Christdemokraten Chiles entwickelte Konzeption einer Volkseinheit unter Führung von Radomiro Tomic, die Christdemokraten, Kommunisten und Sozialisten vereinen sollte. Nachdem die Kommunisten (mit Rücksicht auf die Sozialisten!) diesen Plan abgelehnt hatten, scheiterte er auch innerhalb der Christdemokraten. Siehe Radomiro Tomic, Revolución chilena y Unidad Popular, Santiago 1969. Zur Gegenposition innerhalb der Christdemokratie: Patricio Aylwin, Camino propio. Lo que Chile espera de la Democracia Cristiana, Santiago 1969.

Es entbehrt nicht der Tragik, daß erst nach der faschistischen Wendung der chilenischen Geschichte an einer solchen Allianz gearbeitet wird, die einen 11. September mit all seinen Folgen hätte verhindern können.

5 Zum Prozeß der Herausbildung der Volkseinheit siehe die detaillierte Darstellung von Eduardo Labarca, Chile al rojo, Santiago 1971.

6 Eine Analyse der chilenischen Präsidentschaftswahlen von 1970 findet sich bei Dieter Nohlen, Chile (s. Anm. 3), S. $113 \mathrm{ff}$. Siehe auch Joan E. Garcés, Desarrollo político y desarrollo económico. Los casos de Chile y Colombia, Santiago 1972, hier vor allem S. $283 \mathrm{ff}$.

7 Diese Diskussion setzte gleich nach dem 4. September 1970 ein und verfolgte das Ziel, den geringen Beitrag der Radikalen zum Wahlsieg Allendes nachzuweisen. Rein quantitativ in Stimmenprozenten ausgedrückt mag diese These durchaus richtig gewesen sein. Sie verkannte aber den wirklichen Wert des radikalen Stimmpakets. Ahnlich wie in dem bekannten Beispiel der Wert eines zehn Cent werten Stückes Draht in einer Weltraumrakete, die mehrere Millionen Dollar kostet, wenn die Rakete explodiert, weil das Drahtstück defekt war, nicht einfach gleich zehn Cent ist, so waren auch die radikalen Stimmen mehr wert als eben zwei bis drei Prozent, denn sie verhalfen Allende zum Wahlsieg. 
Die Konzeption eines „zweiten. Weges zum Sozialismus“ berücksichtigte konsequenterweise die soziale Struktur des Parteienbündnisses, das diese Strategie verfolgen sollte. Parlamentarisch und demokratisch, mit den Mitteln des bürgerlichen Staatsapparats sollte der Sozialismus eingeführt werden, ein Sozialismus, der im Bereich der gesamtstaatlichen Willensbildung und in den sozialisierten Unternehmen mit dem demokratischen Element verbunden werden sollte. Hier flossen nicht nur Vorstellungen bürgerlicher Gruppen in die politische Strategie der Volkseinheit ein. Ebenso wichtig war der Beitrag der reformistischen Positionen der Kommunistischen Partei Chiles selbst und vor allem ihrer sozialen Basis, der Industriearbeiterschaft, die durch ihre Beschäftigung im außengerichteten „Monopol"-Sektor der Wirtschaft positiv in das bestehende System integriert ist und Wertvorstellungen und Verhaltensmuster der Mittelschichten angenommen hat ${ }^{8}$. Schließlich anerkannte diese Strategie des sogenannten „chilenischen Weges"9 die sozio-politischen Bedingungen, denen eine revolutionäre Politil in Chile unterworfen war. Sie anerkannte die Verfassungsmäßigkeit als Grundlinie revolutionärer Politik, weil anders die Mittelschichten ins rechte Lager getrieben und vor allem die Streitkräfte als Verteidiger der legalen Ordnung auf den Plan gerufen worden wären. Undogmatisch ging sie von den konkreten Realisierungschancen sozialistischer Politik in einem traditionell demokratisch-parlamentarisch regierten Entwicklungsland aus.

Die Stoßrichtung der im Programm der Volkseinheit ${ }^{10}$ geplanten Maßnahmen war in erster Linie anti-oligarchisch und anti-imperialistisch. Der sozialistische Charakter der propagierten Politik wurde nicht besonders hervorgehoben. Dagegen wurden konkrete Forderungen der lohnabhängigen und marginierten $\mathrm{Be}-$ völkerung betont. Die angekündigten Strukturreformen, die durch dependenztheoretische Argumente begründet wurden, deckten sich weitgehend mit den Vorstellungen breiter Sektoren der Mittelschichten. In den Parteien der Mittelschichten waren während der Regierung Frei modernisierungstheoretische und dualistische Entwicklungsvorstellungen in die Minderheit geraten. Die soziale und wirtschaftliche Entwicklung des Landes wurde immer mehr im Sinne „sozialistischer" Strukturreformen, Ausweitung des staatlichen Sektors, Agrarreform, Kontrolle des Außenhandels und des Auslandskapitals, Planwirtschaft, begriffen. Dort, wo es an theoretischer Einsicht mangelte, nahm das Bekenntnis zu einem Sozialismus, dem ein besonderes Epitethon beigegeben wurde, dem Wort viel von der Furcht, die es vorab den Mittelschichten eingeflößt hatte. So führten besonders die Christdemokraten, seit 1965 stärkste Partei des Landes, ihre Basis, die aufstrebenden unselbständigen Mittelschichten (Techniker, Manager, Bürokraten und Angestellte), Produkt des Modernisierungsprozesses der Wirtschaft in den vierziger Jahren (Volksfrontregierung), an sozialistische Konzeptionen heran („humanitärer, christlicher, kommunitärer Sozialismus“). An dieser Stelle muß auch die Entwicklung der katholischen Kirche in Lateinamerika genannt werden. Be-

\footnotetext{
8 Siehe hierzu Aníbal Pinto, Crítica de una tésis tradicional, in Hernán Godoy, Estructura social (s. Anm. 3), S. $459 \mathrm{ff}$. Dort macht Pinto auch auf die politisch-strategischen Folgen der Verbürgerlichung von Teilen der Arbeiterschaft im extraktiven und verarbeitenden Sektor für eine den Sozialismus

9 Zum chilenischen Weg ${ }^{\alpha}$ siehe Salvador Allende, Chiles Weg zum Sozialismus, Wuppertal 1972; Chile -

9 Zum chilenischen Weg siehe Salvador Allende, Chiles Weg zum Sozialismus, Wuppertal 1972; Chile Allende Gossens, Berlin (Ost) 1973; zur „Übergangswirtschaft ${ }^{\alpha}$ : Gonzalo Martner (Hrsg.), El pensamiento económico del gobierno de Allende, Santiago 1971.

$10 \mathrm{Das}$ Programm der Volkseinheit findet sich in deutscher Úbersetzung in: A. Acquaviva u. a., Das Chile der Volkseinheit, Frankfurt 1971, S. 127 ff.
} 
sonders in Chile bildete sich eine sozial engagierte Priesterschaft heraus, die sich für eine Zusammenarbeit mit den Marxisten aussprach. Das chilenische Episkopat schränkte diese Neuorientierung der Kirche kaum ein ${ }^{11}$. Indem sich die Radikalen der marxistischen Linken wieder annäherten, wurden sie zum Wortführer des "Sozialismus der Mittelschichten“ in den Reihen der Volkseinheit. Ihre programmatischen Vorstellungen formten vor allem den ökonomischen Teil des Basisprogramms, das die Wirtschaft der drei Sektoren, sozialisiert, gemischt und privat, als Modell vorsah.

Das Bündnis zwischen Mittelschichten und Arbeiterschaft blieb aber in vielerlei Hinsicht problematisch. Ein langfristig entscheidender Geburtsfehler lag darin, daß nicht die Partei der neuen, aufstrebenden Teile der Mittelschichten, die sich, wie die Arbeiterschaft, in einer unselbständigen Position befinden, in die Volkseinheit $\mathrm{zu}$ integrieren versucht wurde, sondern die Partei der traditionellen mittelständischen Berufe, die bereits im Niedergang begriffen war ${ }^{12}$. Diese Option war nicht nur von soziologischer Bedeutung, sondern auch institutionen-politisch von großer Tragweite, denn sie verschloß der Volkseinheit die Aussicht, im chilenischen Kongreß eine Mehrheit zu erhalten. Die marxistischen Parteien verbanden sich vor allem deshalb mit den Radikalen, weil sie ihnen gegenüber den politischen Führungsanspruch der Arbeiterklasse im Bündnis glaubten durchsetzen zu können, der sich vor allem in der Auswahl des Kandidaten dokumentierte, der die Volkseinheit anführen sollte.

Gerade in dieser Frage kam es jedoch zu einem ersten Konflikt zwischen der Radikalen Partei, die sich von der Wiederauflage ihrer früheren Bündnispolitik einen neuen Aufschwung erhoffte, und den marxistischen Parteien, der für die Struktur und spätere Entwicklung der Volkseinheit entscheidende Weichen stellte. Aus einer Reihe von Vorkandidaten der einzelnen Mitglieder der Volkseinheit (die Kommunisten hatten den späteren Nobelpreisträger Pablo Neruda aufgestellt) blieben als einzige Alberto Baltra von der Radikalen Partei und Salvador Allende von der Sozialistischen Partei im Gespräch. Die Kommunistische Partei führte die Entscheidung herbei, indem sie den Radikalen nach Wochen der Lahmlegung des sog. Runden Tisches der Volkseinheit mitteilte, daß die Kommunisten keine neue Volksfront akzeptieren könnten, d. h. ein Bündnis, das von den Radikalen angeführt und von Sozialisten und Kommunisten unterstützt wird ${ }^{13}$. Vielmehr sollte "ein Schritt vorwärts in der Geschichte" getan und ein Bündnis auf der Basis einer Klassenallianz gebildet werden, das dieses Mal von einem Vertreter einer "proletarischen Partei“, d. h. von einem Sozialisten oder Kommunisten, geführt werden sollte. Die Radikale Partei beharrte indes auf ihrer Forderung und wich erst, als ihr Kandidat seine Bewerbung zurückzog. Der auf diese Weise erzwungene Verzicht auf die Kandidatur eines Radikalen unterbrach jäh die Perspektive der alten Mittelschichten, nach dem Scheitern des kapitalistischen Entwicklungsweges $^{14}$ nun die sozialistische Entwicklung anführen $\mathrm{zu}$ können. Er

11 Zur Entwicklung der Kirche in Chile siehe den Quellenband: Cristianos por el socialismos: Consecuencia cristiana o alienación política, Santiago 1972.

12 Zur Soziologie des Parteiwesens in Chile siehe vor allem den Beitrag von Enzo Faletto und Eduardo Ruiz, Conflicto político y estructura social, in: Aníbal Pinto u. a., Chile hoy, Mexiko 1970, S. 213-

13 Siehe hierzu Eduardo Labarca, Chile (s. Anm. 5), S. 209 ff. und Régis Debray und Salvador Allende, Der chilenische Weg, Neuwied, Darmstadt, Berlin 1972. Dort hebt Salvador Allende den Fortschritt und Charakter der Volkseinheit gegenüber den früheren Volksfrontregierungen hervor, siehe s. $128 \mathrm{ff}$.

14 Die Radikale Partei hatte bis 1964 die politische Reches des Landes unterstützt, die unter der Regierung des Präsidenten Jorge Allessandri Entwicklungsanstrengungen nach dem Konzept der Allianz für den Fortschritt unternahm. 
verfehlte seine Auswirkungen auf die radikale Anhängerschaft nicht, die sich zu einem Drittel von den CEN-Radikalen ${ }^{15}$ abwandte. Damit war das sozialistische Experiment jedoch noch nicht grundsätzlich in Frage gestellt. Allerdings mußte die von einem Marxisten geführte Volkseinheit davon ausgehen, daß ihre aus den Mittelschichten stammende oder die Mentalität dieser Schichten teilende Anhängerschaft in der politischen Identifikation mit dem linken Parteienbündnis Schwächen besaß und teilweise nur politischen Flugsand darstellte. Es mußte sich in der Praxis erweisen, inwieweit die eingeleitete revolutionäre Politik deren politischer Kultur und deren ökonomischen Interessen entsprechen würde. Deshalb mußte der Führung des Revolutionsprozesses durch die Volkseinheit größte Bedeutung für das Verhalten der Mittelschichten - und damit für das Überleben der Revolution und für die Realisierbarkeit des Sozialismus in Chile überhaupt - zukommen.

\section{Ideologische Differenzen und alternative Strategien}

Einer der wichtigsten Gründe für das Scheitern Allendes liegt in den ideologischen Differenzen und den daraus abgeleiteten politischen Alternativstrategien, die von Anfang an das Bündnis der verschiedenen Parteien und Bewegungen kennzeichneten und im Laufe des Prozesses an Tiefe und Gegensätzlichkeit zunahmen, vor allem durch das verstärkte Einwirken der „Bewegung der revolutionären Linken“, MIR, auf die Sozialistische Partei, den MAPU und die Christliche Linke. Entgegen den Grundannahmen des „chilenischen Weges“ blieben innerhalb der chilenischen Linken zwei ideologische Positionen aufrechterhalten, die mit dem legal-demokratischen Weg unvereinbar und - in praktische Politik umgewandelt - die Volkseinheit zum Scheitern bringen mußten: 1. Der Sozialismus gelingt nur auf einer eindeutigen Klassenbasis; 2. Der Sozialismus läßt sich nur gewaltsam unter Aushöhlung und Zerschlagung des bürgerlichen Staatsapparats einführen.

Wenige Monate nach der Regierungsübernahme durch Allende beschlossen die Sozialisten auf ihrem Parteikongreß in La Serena den ideologischen Kampf innerhalb der Volkseinheit ${ }^{16}$. Sie eröffneten und forcierten den Konflikt mit allen bürgerlichen Gruppen, die teilweise das revolutionäre Programm unterstützten, teilweise ihm ideologisch und/oder interessenpolitisch durchaus nahestanden. Nicht die Durchführung des Programms der Volkseinheit, sondern die Entscheidung in der Frage, welche Klasse Träger der Revolution sei, wurde zu Gegenstand und Maßstab ihrer Politik. Ihre Kampagne schwächte die Radikale Partei und vergrößerte den Graben zwischen Volkseinheit und Christdemokraten, der zunächst - wie die Wahl Allendes mit den Stimmen der Christdemokraten zeigte ${ }^{17}$ - überwindbar schien. Von gleicher Wirkung war die Politik des verschärften Klassenkampfes auf dem Lande, die der MIR leitete und welche die Landarbeiterschaft als revolutionäres Subjekt im chilenischen politischen Prozeß aufwertete, ohne daß sich hier grundlegende ideologische Konflikte ergaben, ob Arbeiter oder Bauern die Revolution zu tragen hätten.

\footnotetext{
15 CEN $=$ Comité Ejecutivo Nacional, Nationales Exekutivkomitee; unter den CEN-Radikalen werden die Radikalen verstanden, die in der historischen Partei verblieben.

$16 \mathrm{Zu}$ den Parteitagsbeschlüssen siehe Julio César Jobet, El partido socialista de Chile, 2. Bde., 2. Aufl., Santiago 1971, S. $169 \mathrm{ff}$.

17 Siehe dazu im einzelnen Dieter Nohlen, Sozio-ökonomischer Wandel und Verfassungsreform in Chile 1925-1972, in VRU, Heft 1/1973, S. 65-85, hier S. $79 \mathrm{ff}$.
} 
Der Mehrheitsflügel der PS und MIR beharrte auch auf der These, daß die Einführung des Sozialismus in Chile wie andernorts nur gewaltsam möglich sei. Sie kritisierten nicht nur die politische Strategie, die dem Programm der Volkseinheit zugrunde lag, also Klassenbündnis und demokratisches Procedere innerhalb der bestehenden Institutionen, sondern propagierten und forcierten die klassenkämpferische Auseinandersetzung durch gewaltsame Aktionen, Aufbau von Gegenmacht, Aushöhlung des Staatsapparates, etc. Dieser Strategie setzten Teile der Opposition, die wie die extreme Linke die parlamentarische Demokratie inhaltlich aufgegeben hatten, den bewaffneten Gegenterror entgegen. Folgenschwerer war jedoch noch der verschärfte institutionelle Widerstand der gemäßigten Opposition der Christdemokraten, wodurch Allende im Parlament abgeblockt wurde ${ }^{18}$.

Die auf den beiden genannten ideologischen Annahmen beruhende zweite Strategie der Revolution in Chile bildete den Grundwiderspruch zur sozialen und politischen Konzeption der Volkseinheit. Sie hinderte die erfolgreiche Durchführung des chilenischen Weges im Rahmen des begrenzten Spielraums, den der friedliche Übergang zum Sozialismus in Chile besaß19. Im Laufe der gleichzeitigen Anwendung beider Strategien, in der weniger kritische Beobachter der chilenischen Szene einen Garanten dafür sahen, daß Chile den Sozialismus aufbauen könne ${ }^{20}$, verschlechterten sich kontinuierlich die Realisierungschancen des chilenischen Weges. Zwar erkannten die Strategen der Volkseinheit, die Kommunisten, allmählich die Unvereinbarkeit der beiden Strategien, waren aber angesichts des self-fulfilling-prophecy-Charakters der Gegenstrategie immer weniger in der Lage, deren Grundaxiome zu widerlegen. Die Empirie, die täglich aussichtslosere Lage des friedlichen Weges, in den ökonomischen Schwierigkeiten am sichtbarsten in Erscheinung tretend, schien der extremen Linken recht zu geben ${ }^{21}$.

\section{Die eingeleitete Wirtschaftspolitik und ihre ökonomischen und politischen Folgen}

Ein weiterer entscheidender Grund für das Scheitern der Volkseinheit bestand in ihren wirtschaftspolitischen Maßnahmen. Ein kurzer Blick auf das Bild, das die chilenische Wirtschaft September 1973 zeigte, sagt uns, daß die Situation nicht anders als chaotisch zu bezeichnen war ${ }^{22}$. Chile wurde von einer Geldentwertung

18 Zur innenpolitischen Polarisierung unter der Regierung der Volkseinheit im einzelnen siehe Dieter Nohlen, Chile (s. Anm. 3) und ders., Feuer unter der Asche. Chiles gescheiterte Revolution, Baden-Baden 1974, S. $105 \mathrm{ff}$

19 Die positiven Voraussetzungen hat Dieter Nohlen in seinem Aufsatz: Warum scheiterte Salvador Allende?, in: aus Politik und Zeitgeschichte, Beilage zur Wochenzeitung Das Parlament, 48/1973, S. 8 ff. herausgearbeitet. Siehe jetzt auch ders., Feuer unter der Asche (s. Anm. 18), S. 38 ff., wo die positiven und negativen, externe und interne Bedingungen gegeneinander abgewogen werden.

20 Dieter Boris, Elisabeth Boris, Wolfgang Ehrhardt, Chile auf dem Weg zum Sozialismus, Köln 1971, S. 281. Wesentlich skeptischer Heinz-Rudolf Sonntag, Revolution in Chile, Frankfurt 1972, ohne allerdings die Problematik der doppelten Strategie recht aufzugreifen.

21 Es ist hier nicht möglich, auf die Diskussion der marxistischen Linken über die strategischen Fehler der Volisseinheit, die nach dem 11. September 1973 einsetzte, näher einzugehen. In der Strategiekontroverse scheint keine der beiden Positionen zur Revision bereit zu sein. Während die MIR-Position hierzulande in Schriften und engagierten Chile-Zirkeln dominiert, dürfte die kommunistische Position eher zur Basis eines neuen linken Parteienbündnisses werden. Siehe u. a. Darcy Ribeiro, Salvador Allende y la izquierda desvariada, in: Postdata, Dez. 1973, S. 7-10; Volodia Teitelboim, Preludio de futuras victorias, in: Revista Internacional, 3/1974, S. 56-60; René Castillo, Chile: enseñanzas y perspectivas de la revolución, Prag 1974. Dagegen: Jaime Faivovich, Pourquoi nous avons échoué, in: Le Monde, 12. 9. 1974, S. 6.

22 Zur wirtschaftlichen Entwicklung 1970-1973 siehe Dieter Nohlen und Klaus Schäffler: Die wirtschaftlichen Gründe des Scheiterns von Salvador Allende, in: Hamburger Jahrbuch für Wirtschafts- und Sozialpolitik, 19/1974, S. 43-65. 
heimgesucht, deren Ausmaß selbst in dem an hohe Inflationsraten gewöhnten Land bisher unbekannt waren. Die Inflation betrug über ein Prozent am Tag. In drei Jahren hatte die Regierung mittels der ihr unterstehenden Zentralbank die umlaufende Geldmenge um 325 Prozent erhöht. Mit dieser Maßnahme sollten die ständig wachsenden Staatsausgaben gedeckt werden, die 1973 nur noch zu etwa 47 Prozent durch Steuereinnahmen finanziert werden konnten. Der verstaatlichte Wirtschaftssektor, dessen Verluste zuletzt fast 70 Prozent seines gesamten Umlaufes betrugen, mußte durch Finanzzuweisungen aufrechterhalten werden. Nicht weniger dramatisch gestaltete sich der Außenhandel. Die von der Regierung Frei übernommenen verfügbaren Reserven in harter Währung in Höhe von 331 Millionen USDollar waren bis auf 41 Millionen aufgezehrt. Die Exporte, die 1970 noch einen Erlös von 1,25 Milliarden Dollar einbrachten, sanken aufgrund in- und ausländischer Negativfaktoren 1972 auf 0,96 Milliarden Dollar und blieben auch 1973 mit 1,19 Milliarden Dollar unter dem Stand von 1970. Im gleichen Zeitraum aber erhöhten sich die Ausgaben für Importe von 1,18 Milliarden Dollar (1970) auf 1,63 Milliarden Dollar (1973). Diese Zunahme ging hauptsächlich auf die Ausweitung der Nahrungsmittelimporte zurück, die durch Produktionsrückgänge landwirtschaftlicher Erzeugnisse bedingt waren. In Verbindung mit der Stagnation der Kupferproduktion trotz erweiterter Abbaukapazität und mit dem Rückgang der Produktion im Industriesektor seit 1972 verminderte sich das BIP pro Kopf der Bevölkerung im zweiten Wirtschaftsjahr der Volkseinheit um etwa 0,9 Prozent. Für das Jahr 1973 weist die Statistik der lateinamerikanischen Wirtschaftskommission der UN einen Rückgang des BIP pro Kopf von 5,7 Prozent aus ${ }^{23}$.

Wie konnte es in weniger als drei Jahren zu einer solchen Situation kommen. Die wesentlichen Gründe dafür liegen in der von der Volkseinheit verfolgten Wirtschaftspolitik, deren fehlerhafte und letztlich verhängnisvolle Grundannahme darin bestand, daß die notwendige soziale und ökonomische Transformation zugleich wirtschaftliche Entwicklung nach sich ziehe. Der begrenzte Handlungsspielraum einer Entwicklungsstrategie, die auf einer grundlegenden Veränderung der bisherigen Strukturen in Wirtschaft und Gesellschaft fußt, und die Bedeutung möglicher Störfaktoren im Transformations- und Entwicklungskonzept wurden sträflich unterschätzt.

Die erste strukturverändernde Maßnahme, die Grundlage der wirtschaftlichen Entwicklung werden sollte, bestand in der Verstaatlichung nationaler und ausländischer Unternehmen. Die erwirtschafteten Profite sollten nicht mehr von privater Seite abgeschöpft oder ins Ausland transferiert werden, sondern in den Dienst der nationalen Entwicklung gestellt werden. September 1973 waren insgesamt mehr als 500 Unternehmen entweder verstaatlicht oder interveniert, einschließlich des großen Kupferbergbaus, der sog. Gran Minería del Cobre, der noch zu 49 Prozent in nordamerikanischem Besitz war und entschädigungslos nationalisiert wurde. Management-Mängel, Streiks, Investitionsabnahmen, Boykotte und verschiedene Unsicherheitsfaktoren vereitelten den Plan, die verstaatlichten Betriebe zu Anführern der nationalen Produktion zu machen. Maßnahmen der enteigeneten US-Firmen gegen das chilenische Kupfer und der Preisverfall des roten Metalls auf dem Weltmarkt taten ein übriges, um die ökonomischen Kosten der Strukturreform in die Höhe zu treiben.

23 Zit. nach El Mercurio vom 9. März 1974. 
Die zweite strukturverändernde Maßnahme vollzog sich im Bereich der Agrarpolitik, die vor allem die Liquidierung des Latifundiums zum Ziel hatte. Diese Aufgabe, die bereits unter der Regierung Frei begonnen worden war, wurde auf der Basis des von der Vorregierung durchgesetzten Agrarreformgesetzes in einem Maße von der Allende-Administration und revolutionären Gruppen auf dem Lande beschleunigt, daß die Enteignung der Großgrundbesitzer praktisch bis Jahresende 1973 abgeschlossen war ${ }^{24}$. Dieser große, historische Erfolg der Revolution wurde jedoch nicht von entsprechenden Maßnahmen zur Steigerung oder auch nur zur Aufrechterhaltung der Produktion begleitet. Der Rückgang der Erträge vor allem im Jahre 1973 nötigte Chile zu immer größeren Devisenausgaben für Nahrungsmittelimporte, die schließlich das Dreifache der Summe betrugen, die das Land für Nahrungsmitteleinfuhren zu Beginn der Allende-Regierung aufbringen mußte.

Der dritte Bereich, in dem sich die Erwartungen der Volkseinheit nicht erfüllten, bestand in den internationalen wirtschaftlichen Beziehungen. Um die Abhängigkeit des Landes vom kapitalistischen Ausland zu reduzieren, setzte sie nicht nur entschädigungslose Enteignungen ausländischer Unternehmen durch, sondern plante auch, die bisherigen westlichen Kreditgeber gegen sozialistische auszutauschen ${ }^{25}$. Beide Maßnahmen schlugen jedoch fehl. Die Nationalisierung verbesserte zum einen nicht die Devisenlage des Landes. Diese verschlechterte sich drastisch durch den Ausfall erwarteter Exporterlöse bei gleichzeitigem erhöhten Importbedarf infolge des Rückgangs der Agrarproduktion. Zum anderen blieb das Ergebnis der Kapitalimporte aus sozialistischen Ländern trotz enormer, aufwendiger Bemühungen um vor allem sowjetische Kredite mager ${ }^{26}$, während - entgegen der weitläufigen Meinung - die Verminderung der Kredite aus dem westlichen Ausland, auf die zurückzugreifen Allende infolge der Verschlechterung der Außenhandelsbilanz gezwungen war, gering war. Allerdings deckten sie nicht im geringsten den zunehmenden Kapitalbedarf Chiles.

Das erste Wirtschaftsjahr Allendes schien zu zeigen, daß die wirtschaftspolitischen Maßnahmen richtig waren. Die Indikatoren waren mit Ausnahme der Investitionen positiv. Auf das Jahr des Konsums und eines beachtlichen Wirtschaftswachstums sollte das Jahr der Investition folgen. Aber im zweiten Wirtschaftsjahr wurden bereits die zentralen Fehler der ökonomischen Politik deutlich. Die Inflation nahm einen galoppierenden Rhythmus an, während gleichzeitig die Produktion stagnierte und teilweise zurückging. Schließlich traten Versorgungsengpässe und schwarze Märkte hinzu. Im Jahre 1973 verstärkten sich noch die wirtschaftlichen Ungleichgewichte, bis sich jenes eingangs geschilderte, chaotische Bild ergab.

Die politischen Folgen der wirtschaftlichen Entwicklung Chiles ließen nicht auf sich warten. Sie mußten für ein Experiment verhängnisvoll sein, dessen Realisierungschance wesentlich darauf beruhte, daß die Wählerschaft ihm mehrheitlich zustimmte, und das heißt, daß Wähler unterschiedlicher sozialer Schichten es trugen.

\footnotetext{
24 Comentarios sobre la situación económica, Primer semestre 1973, Santiago 1973, S. 47. Zur Analyse der Agrarpolitik der Volkseinheit siehe Solon Barraclough u. a.: Chile: Reforma agraria y gobierno popular, Buenos Aires 1973. Des weiteren Wolfgang Weischet, Agrarreform und Nationalisierung des Bergbaus in Chile, Darmstadt 1974.

25 Zur Problematik solcher Neuorientierung siehe Franz Nuscheler, Formen, Wirkweisen und Indikatoren von Abhängigkeit, in Dieter Nohlen und Franz Nuscheler (Hrsg.), Handbuch der Dritten Welt, Band I: Theorien und Indikatoren von Unterentwicklung und Entwicklung, Hamburg 1974, S. $153 \mathrm{ff}$.

26 Dazu im einzelnen Dieter Nohlen und Klaus Schäffler, Die wirtschaftlichen Gründe (s. Anm. 22), S. 60 ff.
} 
Freilich war die wirtschaftliche Entwicklung politisch induziert: von seiten der Volkseinheit, um die politische Machtfrage zu entscheiden ${ }^{27}$. Auch als durch eine „Kriegswirtschaft", eine Wirtschaft organisierten Mangels, die Ungleichgewichte auszugleichen versucht wurde, herrschte unter den OKonomen der Volkseinheit noch immer der machtpolitische Gesichtspunkt vor ${ }^{28}$. Teile der bürgerlichen Anhängerschaft der Volkseinheit waren aber eine Politik mitzumachen nicht bereit, die die extreme Inflation und die allgemeine Wirtschaftskrise als Waffe zur Untergrabung der bürgerlichen Wirtschaft betrachtete, eine Begründung, die uns aus den Anfängen der sowjetischen Wirtschaftspolitik bekannt ist ${ }^{29}$. Mit Alberto Baltra, dem einflußreichen OOkonomen der Radikalen Partei, vollzogen hier weitere Teile der Mittelschichten eine Abkehr von der Volkseinheit ${ }^{30}$.

Von Teilen der in- und ausländischen Opposition wurde eine Politik des ökonomischen Chaos betrieben, um den demokratischen Durchmarsch zum Sozialismus zu verhindern und um als Folge einer in die politische Agonie führenden innenpolitischen Auseinandersetzung langfristig das Militär auf den Plan zu rufen. Die fehlerhafte Wirtschaftspolitik begünstigte die in- und ausländischen Gegner der Volkseinheit, die die Allende-Regierung immer leichter torpedieren konnten. Die intentional auf eine größere Unabhängigkeit Chiles gerichteten Maßnahmen machten Chile immer abhängiger vom Ausland (Kapitalimport) und immer empfindlicher gegenüber den Destabilisierungsmanövern der ausländischen, vor allem nordamerikanischen Unternehmen und des $\mathrm{CIA}^{31}$.

\section{Die Entwicklung des Verhältnisses von Mittelschichten und Volkseinheit}

Die Furcht der Mittelschichten vor den konkreten Maßnahmen, mehr aber noch vor den langfristigen Entwicklungstendenzen der Allende-Regierung, hielt in jedem Moment des sozialistischen Experiments an, immer wieder geweckt durch die bürgerliche Presse, angeführt vom Veteran „Mercurio“32. Allende tat auch zu wenig, um Beruhigung herbeizuführen und dort Sicherheit zu geben, wo ohnehin keine Reformen geplant waren oder strategische Gruppen allein durch das Definitionsvakuum sich gefährdet fühlten. Allende war Taktiker und mied es, sich grundsätzlich festzulegen. Verlangte der Moment eine eindeutige Stellungnahme, so öffnete er sich bald eine Hintertür, um sich die Entscheidung in einer neuen politischen Lage offenzuhalten. Symptomatisch dafür war die Vereinbarung eines Verfassungsstatuts mit den Christdemokraten, welches die Voraussetzung dafür war, daß die Christdemokraten Allende im Kongreß zum Präsidenten wählten ${ }^{33}$. Allende beruhigte damit die Mittelschichten. Wochen später konzedierte er im Interview mit Régis Debray dem anders gesinnten Franzosen, daß diese Übereinkunft „taktisch notwendig“ gewesen sei, um gewählt werden zu können ${ }^{34}$. Als nur

27 Siehe Dieter Nohlen, Chile (s. Anm. 3), S. 170.

28 Siehe das Spiegel-Interview mit dem damaligen Wirtschaftsminister Carlos Matus, Spiegel 43/1972, S. 134.

29 Siehe E. H. Carr, The Bolshevik Revolution 1917-1923. A History of Soviet Russia, Band 2, London 1952, S. 260.

30 Alberto Baltra, Gestión económica del gobierno de la Unidad Popular, Santiago 1973.

31 Dazu Näheres bei Dieter Nohlen und Otto Boye, Sozialreform, Sozialismus, Reaktion. Chile 1964-1970, in: Herderkorrespondenz, 10/1974, S. 530-538.

32 Die ökonomischen Interessen hinter der bürgerlichen Presse analysiert Armando Mattelart, Estructura del poder informativo y dependencia, in: Cuadernos de la Realidad Nacional, 3/1970, S. 37-76.

33 Text des Verfassungsstatuts bei Dieter Nohlen, Republik Chile, in: Verfassungstexte. Beilage zu VRU, Heft $1 / 1973$.

34 Régis Debray und Salvador Allende, Der chilenische Weg (s. Anm. 13), S. 130 f. 
taktischer Schachzug verloren die erweiterten Grundrechte die ihnen von den Mittelschichten beigelegte Bedeutung.

Die Kommunalwahlen vom April 1971 zeigten zunächst einen beachtlichen Erfolg Allendes und der Volkseinheit in der Ausweitung ihrer Wählerschaft. In einer günstigen Konjunkturphase der chilenischen Wirtschaft, in der das Konsumniveau darüber hinaus noch schneller stieg, als es die ökonomische Situation rechtfertigte, gelang es der Volkseinheit, fast 50 Prozent der abgegebenen gültigen Stimmen zu erhalten. Ohne Zweifel war dies politisch der günstigste Moment der Regierung der Volkseinheit, der jedoch zur Entscheidung der politischen Machtfrage nicht genutzt wurde ${ }^{35}$. Am Jahresende 1971 hatte sich die Situation bereits vollends geändert. Die Parteien der Opposition waren inzwischen zur Offensive übergegangen, kritisierten die Regierung heftig wegen der innenpolitischen Unsicherheit (der Wendepunkt für die Christdemokraten war der Mord an Edmundo Pérez Zujovic) und prangerten die sich jetzt einstellenden Krisenerscheinungen in der Wirtschaft an. Der "Marsch der leeren Töpfe" der Frauen des „barrio alto" (besseren Wohngebiets) von Santiago war das erste mitreißende Signal dafür, daß die Mittelschichten sich in eine absolute Opposition zu Allende hineinsteigerten. Fidel Castro, der in jenen Tagen (November 1971) Chile besuchte, sprach in seiner Abschiedsrede im Nationalstadion seine Besorgnis aus: „Wir haben den Faschismus in Aktion gesehen“ ... Etwas später fügte er eine fundamentale Überlegung hinzu: „... der Erfolg oder Mißerfolg dieses außergewöhnlichen Prozesses hängt von der ideologischen Auseinandersetzung und vom Kampf der Massen ab, und er ist abhängig von der Geschicklichkeit, der Kunst und der Wissenschaft der Revolutionäre, zu integrieren, zuzunehmen und die Mittelschichten der Bevölkerung zu gewinnen (Beifall)"36.

Die Mahnung Castros blieb unbefolgt. Die Schwierigkeiten der Revolution mit den Mittelschichten der Bevölkerung vergrößerten sich in immer schnellerem Rhythmus. Der Niedergang der Regierung in diesen Sektoren zeigte sich bei verschiedenen Wahlen ${ }^{37}$, am deutlichsten und folgenschwersten im Oktoberstreik von 1972, den Allende als „Streik des Bürgertums“ bezeichnete. Er umfaßte tatsächlich breite Teile der Mittelschichten, auch der unteren Mittelschicht, die bereits zu dieser Zeit eine „Lösung“ (una salida) forderten, welche einen Sturz Allendes und die Errichtung eines Militärregimes als realer Möglichkeit mit einschloß. Spätestens hier wurde die anti-konstitutionelle Haltung von Teilen der Mittelschichten offenbar, die von der Nationalpartei geführt wurden.

Angesichts des Drucks der Opposition entschied sich Allende zu einem Schritt, den die Christdemokraten verlangt hatten und der allgemein als mutig und einschneidend angesehen wurde, in den Reihen der Volkseinheit aber nicht überall auf Zustimmung traf: Allende trat an die Streitkräfte zwecks Eintritt in die Regierung heran. Diese Maßnahme, deren langfristige Konsequenzen im Moment der Entscheidung selbst nicht abzusehen waren, rettete die Situation und bremste die Entwicklung, die zu einer entscheidenden endgültigen Auseinandersetzung zwischen den sich immer stärker polarisierenden politischen Lagern führte. Man sprach davon, daß Allendes Maßnahme das Land vor dem Bürgerkrieg gerettet habe.

$35 \mathrm{Vgl}$. die Meinung von Fidel Castro, zit. in Dieter Nohlen, Feuer unter der Asche (s. Anm. 18), S. 89.

36 Zit. nach Punto Final, 146/1971, S. 51. Einen Teil der Reden Fidel Castros in Chile enthält diese Ausgabe, einen anderen die Edition: Chile 1971. Habla Fidel Castro, Santiago 1971.

37 Dazu im einzelnen Dieter Nohlen, Chile (Anm. 3), S. 242 ff. 
Die Hauptaufgabe der Militärs in der Regierung bestand darin, die innere Ordnung zu garantieren und für die normale Abwicklung der Parlamentswahlen vom März $1973 \mathrm{zu}$ sorgen, die als wichtig angesehen wurden, da sie eine klarere politische Entscheidung durch die Wählerschaft selbst herbeiführen sollten. Die beiden Aufgaben wurden erfüllt, jedoch das Ergebnis der Wahlen gab mehr Fragen als Antworten auf, da keine Seite die notwendige Stimmenzahl erhielt, um die Waagschale sich endgültig senken zu lassen. In den allzu tagespolitisch bezogenen Analysen der Wahl herrschte die Meinung vor, Allende habe diese Wahlen gewonnen. Doch mit dem Ergebnis war ihm kaum gedient. Zwar konnte die Regierung mit den auf die "Partei der Volkseinheit" entfallenen 44,0 Prozent der abgegebenen Stimmen einen Achtungserfolg erringen, den man ihr - vor allem aufgrund der katastrophalen Wirtschaftslage und der eine Zweidrittelmehrheit für die Oppositionsparteien voraussagenden Wahlpropaganda der Rechten - nicht zugetraut hatte. Das änderte jedoch nichts daran, daß Allende mit dem gleichen Stimmenanteil wie bei den Parlamentswahlen von 1969 institutionell in der Minderheit blieb. Gravierender war noch, daß bei den zweiten allgemeinen Wahlen seit den Präsidentschaftswahlen vom September 1970 die UP-Parteien gegenüber den Kommunalwahlen vom April 1971 sechs Prozentpunkte eingebüßt hatten. Bis zum Ende der Mandatszeit Allendes stand somit fest, daß demokratisch-parlamentarisch die UP-Regierung von der Opposition aus Christdemokraten und Nationalen abgeblockt werden würde, es sei denn, Allende hätte das Regierungsbündnis auf eine neue parteipolitische und soziale Basis zu stellen versucht. Die Grundlagen dafür, eine solche Konsequenz zu ziehen und die Mittelschichten erneut zu umwerben, hatten sich jedoch gegenüber 1970/71 wesentlich verschlechtert.

Diese politische Absicht lag aber auch fern der nach dem als Wahlsieg interpretierten Wahlausgang angestellten taktischen und strategischen Überlegungen der Volkseinheit ${ }^{38}$. Selbst Allende, immer unzufriedener mit der extremistischen Entwicklung seiner eigenen Partei, glaubte die Situation gekommen, die politischen Bedingungen vor dem Oktoberstreik des Vorjahres wiederherzustellen und entließ die Militärs aus der Regierung. Der Revolution sollte wieder Gas gegeben werden. Doch hatte nicht diese Politik unter dem Vorrang des Klassenkampfes das Land sechs Monate zuvor an den Rand des Bürgerkriegs geführt? War anzunehmen, daß nach den Märzwahlen jetzt bessere Voraussetzungen für eine Durchsetzung sozialistischer Politik nach der doppelten Strategie bestanden? Mitnichten! Auf allen Ebenen spitzten sich nun die Gegensätze zwischen Regierung und Opposition $z^{39}$. Bewußt oder unbewußt wurde von allen Beteiligten einer gewaltsamen Lösung des Konflikts in die Hände gespielt ${ }^{40}$. Diese Entwicklung mußte den Streitkräften eine immer größere Bedeutung für den Ausgang des chilenischen sozialistischen Experiments einräumen.

38 Vgl. die Analyse der Wahlen von Luis Corvalán in: El Siglo, vom 25. April 1973.

39 Dazu ausführlich Dieter Nohlen, Feuer unter der Asche (s. Anm. 18), S. $114 \mathrm{ff}$.

40 Vgl. Radomiro Tomic: „Es wäre ungerecht zu verneinen, daß die Verantwortlichkeit einiger größer sei als die anderer, aber die einen mehr, die anderen weniger, alle tragen wir dazu bei, die chilenische Demokratie zu zerschlagen“, in einem öffentlichen Schreiben an General Carlos Prats vom 29. April 1973, anläßlich des Rücktritts des Generals von seinem militärischen Amt und als Minister. 


\section{Die chilenischen Streitkräfte: Funktion im politischen System und Rolle im Revolutionsprozeß}

Die chilenischen Streitkräfte ${ }^{41}$ sind immer ein gewichtiger innenpolitischer Faktor gewesen. Die langen Perioden ziviler Politik haben diese Tatsache, die auch von marxistischer Seite unterschätzt wurde ${ }^{42}$, in den Hintergrund treten lassen. Erst der 11. September 1973 hat die Bedeutung des Militärs im politischen Prozeß Chiles schlagartig erkennen lassen.

Gewiß, die Zahl der unmittelbaren Interventionen der chilenischen Streitkräfte in die Politik sind an Zahl gering, doch zeugen sie von hoher Effektivität und politischer Tragweite. Betrachten wir nur unser Jahrhundert, so ist die Einführung der parlamentarischen Demokratie nach präsidentiellem Muster und soziologisch die Etablierung der Herrschaft der Mittelschichten das Ergebnis zweier einschneidender Eingriffe des Militärs in die Politik in den Jahren 1924 (gegen die Oligarchie) und 1931 (gegen die nachdrängende revolutionäre Arbeiterschaft, Zerschlagung der ersten sozialistischen Republik ${ }^{43}$ ).

Trotzdem verfestigte sich in den Mittelschichten eine Anti-Gorilla-Haltung, die u. a. auch ein Motiv der sozialen Politik der von den Mittelschichten getragenen Reformregierungen war. Die Integration der Massen der Landarbeiter und der randständischen Bevölkerung in das politische System, welche die Christdemokraten einleiteten, galt der sozialen Befriedigung von Gruppen, die ein revolutionäres Potential darstellten, und welche mit der Überwindung revolutionärer Gefahren auch das Ziel verfolgte, das Militär als innenpolitischen Faktor weiter zu neutralisieren $^{44}$. Wie weit die Christdemokraten in dieser Hinsicht gekommen zu sein glaubten, wurde deutlich, als sie die berechtigten ökonomischen Interessen der Militärs negierten und im Jahre 1969 das Tacnazo-Abenteuer des Generals Viaux Marambio heraufbeschworen ${ }^{45}$. Im letzten Regierungsjahr Freis versuchte General Schneider, der neue Generalstabschef der Streitkräfte, die Disziplin wiederherzustellen und die Streitkräfte auf die Nichtintervention in den politischen Prozeß festzulegen, solange die politischen Gruppen die bestehende Verfassung achteten. Die sog. Schneider-Doktrin, auf die Allende später immer wieder

41 Auf eine Analyse der chilenischen Streitkräfte hinsichtlich ihrer Stärke, ihrer technischen Ausrüstung, ihres Aufbaus und ihrer Entwicklung muß hier aus Raumgründen verzichtet werden. Einige Angaben finden sich bei Dieter Nohlen, Chile (s. Anm. 3), S. $281 \mathrm{ff}$., dort auch weiterführende Literatur. Nach dem Putsch und zu seiner Verteidigung erschien das Sammelwerk von Pablo Baraona u. a., Fuerzas Armadas y seguridad nacional, Santiago 1973.

$42 \mathrm{Zu}$ den spärlichen Äußerungen zählen Salvador Allende, Nuestro Camino al socialismo - La via chilena, Santiago 1971, S. $125 \mathrm{ff}$.; Allende betonte immer wieder seine Überzeugung von der Verfassungstreue der chilenischen Streitkräfte; siehe hier auch das Interview mit Régis Debray (s. Anm. 13), S. 98. Des weiteren Eduardo Labarca, Corvalán 27 horas. El PC chileno por fuera y por dentro, Santiago 1972 S. $116 \mathrm{ff}$. Größere Bedeutung kommt heute der marxistischen Studie von Alain Joxe, Las fuerzas armades en el sistema político chileno, Santiago $1970 \mathrm{zu}$.

43 Aus der ersten Republik überkam das Dekret Nr. 502, das der Regierung der Volkseinheit als gesetzliche Grundlage für die Verstaatlichungspolitik im Industriesektor diente. Siehe Eduardo Novoa Monreal, Vias legales para avanzar hacia el socialismo, in: Mensaje 179/1971, S. 84-90.

44 Eine solche Politik wird auch dort verfolgi, wo die Schwäche ziviler Regierungen inzwischen die Militärs an die Regierung gebracht haben. So betreiben die peruanischen Militärs eine Politik der Neutralisierung der sozialen Basis aufständisch-revolutionärer Bewegungen. Entwicklungspolitik wird zur Funktion der inneren Sicherheit des Landes. Siehe dazu die verschiedenen Beiträge in J. Matos Mar u. a., Perú hoy, Mexico 1970. Die Volkseinheit konnte politisch nicht das gleiche Ziel verfolgen, denn das hätte den revolutionären Charakter ihrer gesellschaftlichen Zielvorstellung aufzugeben bedeutet. Eine in diese Richtung der radikalen gesellschaftlichen Strukturreform zielende Politik mußte Mittelschichten und Militär zu neutralisieren versuchen.

45 Anzumerken ist hier, daß die Sozialistische Partei Chiles in Roberto Viaux Marambio seinerzeit einen Caudillo sah, der der chilenischen Geschichte eine Wendung in sozialistischer Richtung geben könnte. Sie begrüßte deshalb den Tacnazo. Viaux Marambio wurde unter Allende von einem chilenischen Gericht zu einer Gefängnisstrafe von zwei Jahren verurteilt, die praktisch durch die Untersuchungshaft bereits verbüßt war. Zu den Hintergründen des Attentats auf General Schneider siehe Florencia Varas, Conversaciones con Viaux, Santiago 1972. 
rekurrierte, wies den Streitkräften die nicht unwichtige verfassungspolitische Rolle zu, über die Einhaltung der Verfassung zu wachen. Jede Politik, die sich im Rahmen der Verfassung entwickelte, genoß den Schutz der Streitkräfte. Eine Politik hingegen, die unter Umgehung der Verfassung oder gegen sie gerichtet voranschritt, mußte Sanktionen der Streitkräfte befürchten. In der Doktrin Schneider lagen somit Chancen als auch Begrenzungen einer sozialistischen Politik in Chile.

Wir haben bereits oben dargelegt, daß die Definition und Strategie des chilenischen Weges zum Sozialismus der sog. "correlación de fuerzas" in Chile, d. h. den gegebenen gesellschaftlichen und verfassungspolitischen Kräftekonstellationen entsprachen. Die Streitkräfte stellten einen zentralen Pol in diesem Kräftefeld dar. Der demokratische Weg zum Sozialismus, der sich seinen Prämissen gemäß entwickelte, fiel unter die Verfassungsgarantie der Streitkräfte. Das zeigte sich sehr bald nach dem 4. September 1970, als die Streitkräfte gegen alle Instigationen und später Anfeindungen durch inländische Gruppen (Oligarchie, Nationalpartei) und ausländische Mächte (bürgerliche Weltpresse, nordamerikanische Konzerne und CIA) ihre verfassungsloyale Haltung aufrechterhielten. Das Attentat auf General Schneider bekräftigte sie sogar noch ${ }^{46}$. Weil dem so war, konnte Allende späterhin General Schneider zur Symbolfigur des verfassungskonformen Verhaltens der Streitkräfte erheben.

In den Streitkräften stand der Volkseinheit von Anfang an die eigentliche Gegenmacht im Staate gegenüber. Das von der Opposition beherrschte Parlament im gewaltenteilig angelegten Verfassungssystem konnte nur die starke Rolle spielen, weil die Streitkräfte die Stellung des Kongresses garantierten. Indem die Präsenz der Streitkräfte die Allende-Regierung an ein verfassungsmäßiges Vorgehen band, konnte die Opposition die gegebenen Oppositionschancen im bestehenden politischen System voll nutzen. Der doppelten Strategie der extremen Linken versuchte die Opposition ebenfalls über das Militär beizukommen. Durch den Oktoberstreik 1972 zwang sie Allende, Militärs in die Regierung aufzunehmen, die aus der Regierung heraus die UP-Administration an Recht und Gesetz binden und die Aktivität der Linksextremisten in Schach halten sollten.

Durch diese Maßnahme wurden die Streitkräfte freilich immer stärker in den politischen Prozeß involviert, den Chile durchlebte. Die politische Inpflichtnahme des Militärs durch die Regierungsbeteiligung erhöhte die internen Gegensätze und den Widerspruch zwischen politischer Funktion und sozialer Herkunft der Streitkräfte. Zwar versuchte General Carlos Prats Gonzales, Innenminister und zugleich Oberbe. fehlshaber der Streitkräfte, die Mission des Militärs als (partei-)unpolitisch zu charakterisieren ${ }^{47}$. Doch in der wachsenden innenpolitischen Polarisierung konnte die für den Ausgang des sozialistischen Experiments entscheidende Institution eine solche Rolle nich $\tau$ spielen. Sie gab den Ausschlag zugunsten der Volkseinheit, wenn ihre Vertreter in der Regierung auf der Ebene der sog. mandos medios (mittleren Führungskräfte) die Politik der Sozialisierung auf ungenügender gesetzlicher Grundlage oder gar gegen bestehende Gesetze zuließen. Die Streitkräfte entschieden darüber, ob sie das Gesetz über den Waffenbesitz anwandten oder nicht und damit, ob sie der Bewaffnung der Extremisten rechts und links freien Lauf ließen oder den Aufbau illegaler Kampfgruppen verhinderten. Das im Oktober 1972 verabschiedete

\footnotetext{
46 Siehe Dieter Nohlen, Die chilenische Christdemokratie nach den historischen Wahlen, in: Civitas 10/ 1971, S. $257 \mathrm{f}$.

47 Siehe hierzu das Interview von General Carlos Prats im Ercilla, Nr. 1950/1972, S. 9 ff.
} 
Gesetz führten die Streitkräfte erst nach dem 29. Juni 1973 aus, als der Putschversuch des Obersten Souper sowohl die interne Zerrissenheit der Streitkräfte aufgedeckt als auch den Grad der Bewaffnung und Verteidigungsbereitschaft der Arbeitermilizen u. a. sichtbar gemacht hatte. Durch die nun einsetzenden rigorosen Aktionen gegen die im Aufbau befindliche „Bewaffnete Linke“ gaben die Militärs den Ausschlag zuungunsten der Volkseinheit, eine Politik, die nach der Ablösung von Carlos Prats als Oberbefehlshaber geradlinig zum 11. September 1973 hinführt. Das erkannte der MIR durchaus richtig, als er in einer der letzten Nummern des „Punto Final“ vor dem Putsch die Parole ausgab: „Das Wort hat der Genosse Mauser"48. Der politische Prozeß Chiles, in welchem die Streitkräfte zunächst nur als potentieller Machtfaktor auftraten, involvierte zunehmend das Militär und führte als Folge der Verschärfung der sozialen Konflikte zu einer erneuten Übereinstimmung von politischer Funktion und sozialer Herkunft der Streitkräfte. Der Verlauf des Revolutionsprozesses schuf die Voraussetzungen für den Putsch der Streitkräfte.

Wir wollen das Verhältnis von Sozialismus und Militär in Chile abschließend thesenartig zusammenfassen und dabei auch auf die Frage, die der Titel unseres Beitrags stellt, eine Antwort geben. Bestand die Möglichkeit einer sozialistischen Revolution nach dem Programm der Volkseinheit in Chile? Wir fragen damit nicht nach der sozialistischen Stringenz des Programms des linken Parteienbündnisses, sondern nach der Möglichkeit, dieses Programm angesichts der bestehenden sozioökonomischen und politischen Machtverhältnisse durchzusetzen.

These 1: Ein demokratischer Weg zum Sozialismus wurde durch die Präsenz einer bürgerlichen Armee, einer den Mittelschichten des Landes entstammenden und auf die Einhaltung des bürgerlichen Institutionensystems verpflichteten Streitmacht nicht generell in Frage gestellt. Die Schneider-Doktrin war im ersten Regierungsjahr von Allende, in welchem einschneidende Strukturreformen erfolgten, richtungweisend und bestimmend für das Verhalten der Militärs gegenüber der Volkseinheit. Die Beteiligung der Streitkräfte an der Regierung ab Oktober 1972 wurde zwar von der Opposition erzwungen. Sie zeugt indes von der Disposition der Streitkräfte, eine verfassungsgemäß zustande gekommene Regierung zu stützen und den extrem zugespitzten gesellschaftlichen Konflikt einer demokratischen Lösung zuzuführen.

Konnten andere Wege zur Einführung des Sozialismus in Chile beschritten werden als jener, den das Programm der Volkseinheit aufzeigte und dem die Wählerschaft in der Wahl Allendes das Mandat einer relativen Mehrheit, der chilenische Kongreß das Mandat einer absoluten Mehrheit erteilt hatte?

These 2: Nicht-demokratische Strategien zur Einführung des Sozialismus in Chile mußten die Sanktion der Streitkräfte fürchten. Die Realisierungschancen einer nicht demokratisch induzierten und durchgeführten Revolution ${ }^{49}$, einer sozialistischen Po'ititi unter Umgehung oder gegen die bürgerliche Verfassung,

48 Punto Final, Nr. 190 vom 14. August 1973.

49 Es ist offensichtlich, daß der hier verwandte Revolutionsbegriff die Frage der Gewaltsamkeit des Prozesses nicht zum entscheidenden Kriterium erhebt. Zur Begriffsbestimmung siehe Dieter Nohlen, Chile (s. Anm. 3), S. $17 \mathrm{f}$.

50 Hier schließen sich die Autoren der Meinung der führenden Wirtschaftsfachzeitschrift Chiles, des Panorama Económico an; deren Redakteure betonten immer wieder, daß die chilenische Revolution friedlich bzw. der Weg zum Sozialismus in Chile demokratisch verlaufen müsse, oder es gebe ihn nicht. 
waren von Anfang an gering50. Alle Überlegungen, die die Ursachen des Scheiterns von Allende auf das Problem reduzieren, daß die chilenische Revolution nicht dem wissenschaftlichen Sozialismus und ihm entlehnter Handlungsanleitungen gefolgt sei, verkennen die Grundtatsache, daß die Voraussetzungen für das Gelingen eines gewaltsamen sozialistischen Umsturzes in Chile denkbar schlecht waren.

Überlegungen, die hervorheben, daß unter der Volkseinheit zuwenig getan worden sei, um die Chancen für eine erfolgreiche Anwendung der revolutionären Durchbrechungsstrategie (Massenmobilisierung, Aushöhlung des bürgerlichen Staatsapparats, Aufbau von Arbeiter- und Bauernmilizen, Infiltration der Streitkräfte etc.) zu verbessern, berücksichtigen nicht, daß die in diese Richtung unternommenen Anstrengungen den demokratischen Weg hinderten und schließlich versperrten, bevor sie das Gelingen der zweiten Strategie sicherstellen konnten.

These 3: Der Militärputsch vom 11. September galt weniger Allende und dem Programm der Volkseinheit, als vielmehr der politischen Strategie der Linksextremen. Er galt Allende und der Volkseinheit insofern, als der demokratisch-parlamentarische Weg zum Sozialismus nach fast drei Jahren AllendeRegierung aussichtslos geworden war und statt durch ein demokratisches Votum der Wählerschaft durch die MIR-Strategie abgelöst zu werden drohte. Diese Strategie, die in ursächlichem Zusammenhang mit dem Scheitern des chilenischen Weges zum Sozialismus steht, gewann nach den Märzwahlen von 1973 innerhalb der marxistischen Linken die Oberhand. Indem die chilenische Revolution ihre demokratische Komponente - die Voraussetzung ihres Gelingens - einbüßte, wurde der Weg frei für gewaltsame Lösungen, die die Extremisten rechts und links unablässig forderten und herbeizuführen trachteten. Die Gewalt der Militärs kam der Gewalt der Linksextremisten zuvor.

Die drei Thesen dürfen nicht dahingehend mißverstanden werden, als rechtfertigten sie das Eingreifen der Militärs noch gar die brutale Diktatur, die sie in Chile errichteten ${ }^{51}$. Die Absicht ist vielmehr, auf die Fehler der Linken in Chile hinzuweisen, die zum 11. September 1973 geführt haben. Dabei wird nicht verkannt, daß auch andere politische Kräfte des In- und Auslandes diese Entwicklung provozierten. Auch wenn dies in Rechnung gestellt wird, bleiben die Fehler der Volkseinheit für das Scheitern Allendes entscheidend. Sie war nicht fähig, den Revolutionsprozeß entsprechend seinen Erfolgprämissen zu führen, ja der chilenische Weg zum Sozialismus in der Konzeption des Programms der Volkseinheit wurde recht eigentlich nicht beschritten. Als die Militärs putschten, war der zweite Weg zum Sozialismus bereits gescheitert ${ }^{52}$. Was im August 1973 an Entwicklungsmöglichkei-

51 Siehe dazu den Beitrag der Autoren in Herderkorrespondenz, (s. Anm. 31) und auch Dieter Nohlen, Elend statt Chaos, in: DIE ZEIT Nr. 38 vom 13. September 1974.

52 Demgegenüber hält Albert Schenk "die These von einem offenkundigen Scheitern bereits vor dem Putsch durch kein Argument abgesichert". So in: Ist der demokratische Sozialismus in Chile gescheitert?, in: Investitionskontrolle gegen die Konzerne, hrsg. von Manfred Krüper, Reinbek bei Hamburg 1974, S. 115-179, hier S. 177. Schunk bringt für seine Gegenthese, die eine allzu vordergründige Apologie des demokratischen Sozialismus versucht, kein stichhaltiges Faktum vor (und begeht auch den Irrtum, die Unterzeichnung des Waffengesetzes durch Allende wenige Wochen vor September 1973 anzusetzen, die im Unterzeichnung des Waffengesetzes durch Allende wenige Wochen vor September 1973 anzusetzen, die im
Oktober 1972 auf Druck der Streitkräfte und der Christdemokraten erfolgte) und unterschätzt die innenpolitische Ausweglosigkeit der Allende-Regierung nach den Märzwahlen von 1973. Siehe dazu ausführlich Dieter Nohlen, Feuer unter der Asche (s. Anm. 18), S. $120 \mathrm{ff}$. 
ten verblieb, war nicht sehr verschieden von der Alternative, die der MIR bereits propagierte, als ein dritter Weg noch offen stand: Faschismus oder Sozialismus (Diktatur der Arbeiter und Bauern) ${ }^{53}$. Mittelschichten und Militär gaben den Ausschlag.

53 Diese Alternative wählte H. R. Sonntag auch für seine neueste Edition lateinarnerikanischer Autoren Lateinamerika: Faschismus oder Revolution, Berlin 1974, die uns erst nach Abschluß des Manuskripts zuging. Ruy Mauro Marini und H. R. Sonntag nehmen in ihren Beiträgen zu Chile Stellung. Trotz verschiedener Interpretationsunterschiede zwischen den genannten Autoren und uns verdienen zwei Ubereinstimmungen hervorgehoben $\mathrm{zu}$ werden. Marini sieht den "chilenischen Weg zum Sozialismus" als unter spezifischen gesellschaftlichen Bedingungen gültige Strategie an; Sonntag besteht darauf, "daß der chilenische Prozeß keineswegs von Anfang an zu dem Ende verurteilt war, das ihn dann erreichte ..., auch das Problem des Militärs hätte eine Lösung finden können“, S. 84 und 153. 
Was the counterrevolution inevitable? Middle sectors and armed forces in Chile

\section{By Dieter Nohlen and Otto Boye}

The contribution of the two authors, who lived in Chile during the Allendegovernment, deals with the question, whether the Chilean way to socialism had a chance to be successful. It is argued that the democratic road to socialism was a valid concept for Chile. Its success depended mainly on the capacity of the socialist forces to attract middle sectors by responding to their claims and to their political attitudes. In elaborating the program of the Unidad-Popular-Government, the Chilean left and above all the Chilean Communist Party showed a clear conception of this necessity. The program favoured a policy leading to the neutralization of middle sectors and the armed forces.

The authors show how, during the Allende government, this conception was disregarded and finally completely abandoned by the Chilean left supporting the socialist government, so that Allende lost all possibilities to win general elections. Two factors led to this impass: The ideological and strategical contradictions within the left itself and the unbalanced economic policy, causing economic distortions. These factors favoured the unconditional opposition of groups within and outside Chile, as well as left wing socialists, proposing and preparing a violent solution of the political conflict. The armed forces intervened, when the Chilean way to socialism had lost any chance.

\section{Comunidad Industrial - an attempt to create a new structure and control of property in the peruvian industry}

\section{By Mechthild Minkner}

With the General Industries Law (D. L. 18350 of 1970) the peruvian revolutionary government initiated several reforms in the industrial sector, such as reservation of basic industries for public enterprises, promotion of industrial products according to a scale of priority, new regulations of foreign investment rules and participation of workers in industrial firms by the Comunidad Industrial.

The paper deals with the fundamental features and the development of the Comunidad Industrial as an instrument for creating the reformed private sector industrial firm. At first, on the base of the two main laws (D. L. 18350 and D. L. 18384) and the additional regulations the objectives, the operating structure and the organs of the Comunidad Industrial are analyzed. In concept, the Comunidad Industrial is a way for increasing both capital formation and production as well as for achieving conciliation between labor and capital. It establishes the participation of workers not only in profit and property but also in management. After that, guided by statistics, the range and the consequences of the concept are explained. As a matter of fact, the Comunidad Industrial up today has intensified the contradictions between labor and capital. In order to boycott the Comunidad Industrial the entrepreneurs are using different tools extending from deflating net income to fraudulent bankruptcy. Regarding the employees the realization of the Comunidad Industrial is mostly delayed by lack of educational background and instruc- 